\title{
Labyrinthe
}

23 | 2006 (1)

Quatre chercheurs de l'étranger

\section{« Mémoires du Cambodge »}

Thèse de doctorat, sous la direction d'Hélène Cixous, soutenue au Centre de recherches en études féminines de Paris VIII, 1999.

\section{Ashley Thompson}

\section{(2) OpenEdition}

\section{Journals}

Édition électronique

URL : http://journals.openedition.org/labyrinthe/1153

DOI : $10.4000 /$ labyrinthe.1153

ISSN : 1950-6031

Éditeur

Hermann

\section{Édition imprimée}

Date de publication : 15 janvier 2006

Pagination : 15-26

ISBN : 2-9526131-0-9

\section{Référence électronique}

Ashley Thompson, « « Mémoires du Cambodge » », Labyrinthe [En ligne], 23 | 2006 (1), mis en ligne le 23 juillet 2008, consulté le 19 avril 2019. URL : http://journals.openedition.org/labyrinthe/1153 ; DOI : 10.4000/labyrinthe.1153 


\section{« MÉMOIRES DU CAMBODGE »*}

Ashley THOMPSON

\section{D'une mémoire l'autre ${ }^{1}$}

C'est à la généreuse invitation de Labyrinthe que je reprends ici l'introduction à des «Mémoires du Cambodge», consacrées à ce que l'on appelle désormais l'«Époque moyenne» de l'histoire du Cambodge, période historique qui s'étend de la désagrégation de l'Empire angkorien vers le XIV ${ }^{\mathrm{e}}$ siècle jusqu'aux débuts de la colonisation française au XIX siècle. La thèse essaie de dégager un certain nombre de dispositions culturelles, à la fois physiques, «naturelles » et « mystiques » ou symboliques, qui semblent répondre à des urgences particulièrement pressantes à l'Époque moyenne, sans toutefois se limiter à celle-ci. Il y va notamment du devenir mémorial d'un héritage monumental, de la capitalisation d'un potentiel de messianicité, d'un travail de guérison qui passe par un rapport particulier au corps de l'autre et par les apories irréductibles de la généalogie: là où passé et à-venir se conjuguent au présent.

L'Époque moyenne constitue une période historique que l'on peut dire délaissée par l'Histoire, d'où l'intérêt à mes yeux, et surtout aujourd'hui, de s'y attarder, en particulier pour ce numéro: l'étude de cette époque dont on a apparemment si peu de traces exige en effet une méthodologie qui donne du soubresaut aux disciplines universitaires traditionnelles et la question des sources, comme mémoire, comme archives à interpréter, est au centre de cette recherche. Ces «Mémoires du

\footnotetext{
* Thèse de doctorat, sous la direction d'Hélène Cixous, soutenue au Centre de recherches en études féminines de Paris VIII, 1999.

1. Il s'agit du titre d'une discussion entre Hélène Cixous et Jacques Derrida dans la soirée du 23 juin 1998 au grenier du château du Centre culturel international de Cerisy-la-Salle lors du colloque « Hélène Cixous : croisées d'une œuvre ». Je me permets de l'emprunter ici afin de relancer quelques questionnements soulevés ce soir-là autour de la mémoire, ainsi que pour indiquer les autres sources de mes «propres» mémoires du Cambodge: l'œuvre d'Hélène Cixous, poète de la plus extrême contemporanéité millénaire, et celle de Jacques Derrida, son autre philosophique.
} 
Cambodge » engagent une réflexion double, d'une part sur un ensemble de sources très diverses en termes formels et linguistiques (inscriptions en khmer, pali et sanskrit, manuscrits et légendes orales en khmer, temples, statues et bas-reliefs, expériences ethnographiques anciennes et récentes) et d'autre part sur le statut de la source et le rapport de l'historien, comme interprète de la mémoire, avec les sources. Tout en s'inspirant des apports et des méthodologies de nombreux domaines classiques (histoire, épigraphie, iconographie, ethnographie...), il s'agit d'entamer une approche lectrice de ce matériau très riche, approche qui se distingue par une extrême attention prêtée à la textualité de tous ces « textes ».

Ce questionnement de sources, déjà interdisciplinaire, me semble se prêter aujourd'hui, en débordant le cadre de la thèse doctorale et l'étude d'une ère historique et d'une aire culturelle et géographique finalement très limitées, à une réflexion générale en cours, «mondiale » ou mondialisée, et dont on a de nouveaux indices tous les jours: une réflexion, ou en tout cas, un affairement discursif sans précédent, qui se préoccupe, du moins en apparence, du statut de la mémoire. Ainsi, dans la mesure où elle suscite et facilite une interrogation profonde sur la place, sur le rôle parfois, de l'épouvantail de l'Histoire à notre époque, disons, pour ce qui nous concerne et pour aller vite, chez les postcolonisés comme chez les postcolons, l'étude de l'Époque moyenne cambodgienne participe aux débats actuels les plus pressants. Il y va des innombrables tentatives d'imposer aux Cambodgiens certaines pratiques et formes de mémoire, une certaine idéologie mémoriale et donc une idéologie de la culture « ellemême », véhiculées dernièrement et de la manière la plus visible par le Tribunal international qui vient de se constituer à l'initiative nécessaire, certes, mais si trouble, des Nations unies pour juger les crimes des Khmers rouges. En France même, dans le contexte actuel, on ne saurait ignorer la résonance d'un tel projet sur les débats politiques, médiatiques et universitaires portant sur l'histoire coloniale, et sur tout ce qui se dit ou se légifère, s'écrit ou ne s'écrit pas sur ses « rôles », jadis, ailleurs, ou ici maintenant. Ce n'est pas un hasard si l'étude plus ou moins sérieuse de l'Époque moyenne cambodgienne ne s'est lancée qu'à la suite de l'Indépendance: les temps s'y prêtaient. Les recherches entreprises depuis plus d'un siècle sous la direction des organes dits scientifiques français s'étaient focalisées sur les artefacts physiques, objectifs, de la glorieuse époque ancienne; alors que ceux-ci sont loin d'avoir dit leur dernier mot, elles ont fini, par suite des succès inespérés qu'elles ont obte- 
nus, par effriter les bases de leur propre nécessité. Une deuxième génération de chercheurs a donc pris le relais. Plusieurs travaux de thèse réalisés au cours des années 1950-1980 ont permis de déblayer le terrain de l'Époque moyenne. On y verra des images en miroir: si les colons se sont identifiés à la gloire d'Angkor, les postcolonisés se sont retrouvés dans les décombres de l'Empire. Que ce mouvement dit scientifique soit mené par les chercheurs cambodgiens n'étonnera pas, mais qu'à sa véritable tête se trouve une Cambodgienne ${ }^{2}$ dit la puissance de la résistance qu'il se devait d'offrir à la colonisation de l'histoire.

Pour ces chercheurs de l'Époque moyenne, la quantité de sources et leur nature n'étaient plus les mêmes que pour leurs prédécesseurs scientifiques, mais les méthodes de recherche n'en étaient pas moins dans la lignée de tout ce qui avait été établi par le passé. On a analysé les inscriptions, les chroniques des Khmers et des étrangers au Cambodge, les manuscrits littéraires, religieux et juridiques, et la production artistique. Il s'agissait d'un formidable effort destiné à combler le vide, le colossal déficit de connaissances entre l'époque ancienne et l'époque moderne. Désormais et grâce à ces pionniers se prépare une nouvelle génération dans la vie des études khmères - et au-delà, où les voies ouvertes et encore à ouvrir se tracent différemment. Souvent de biais, à travers les frontières des domaines de recherche traditionnels. De tels trajets impliquent des risques qui ne sont pas nouveaux: il faut respecter l'intégrité de l'ancienne discipline traversée et en même temps trouver l'élan de la traversée elle-même qui doit ignorer visas et douanes. En faisant une lecture minutieuse de divers phénomènes qu'il faut bien appeler « khmers » tout en cherchant à voir dans ceux-ci leurs continuités et ruptures par rapport à d'autres mondes, ce travail s'engage, tant bien que mal, sur ces voies risquées.

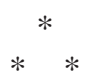

Étudier l'Époque moyenne est un choix, si c'en est un, qui m'aura choisie, un choix en somme plus que surdéterminé et qui reste pour moi, peut-être davantage encore que l'époque elle-même, en grande partie obscur. Déterminé d'abord sans doute par ma propre histoire depuis la

2. Saveros Pou (Lewitz). 
plus petite enfance et dont l'anamnèse ne livrera jamais tous les secrets. Déterminé ensuite par l'histoire récente du monde, et ce à plus d'un titre. Avant tout il y a la décolonisation ou l'Indépendance. L'Époque moyenne cambodgienne, nous ne saurons l'oublier, est celle d'un empire ou d'une puissance coloniale après sa « décolonisation ». C'est sur le devenir de cet « après » que s'interroge cette thèse.

Or, le monde d'aujourd'hui est à la fois « postcolonial » et en voie de mondialisation. Ce n'est là qu'apparent paradoxe. Le dialecticien y verra une certaine suite historique: la mondialisation, ou les divers phénomènes auxquels on prête ce nom, n'a été possible que dans un monde placé sous le signe du postcolonialisme. Mais, du coup, on peut craindre, en effet, que la mondialisation ne cache de nouvelles formes de colonisation, c'està-dire une postcolonisation. On pourrait même soupçonner les études postcoloniales d'être une ultime ruse, imaginée sur le tard par les postcolons pour empêcher, par d'autres moyens, les postcolonisés de reprendre l'avantage. Mais dans les décombres de ce monde non-encoremondialisé se présentent de nouvelles possibilités de recherche, de nouvelles approches ou perspectives possibles, de nouvelles positions et superpositions qui enjambent plusieurs frontières à la fois.

Nous vivons donc dans une sorte d'époque moyenne de la pensée occidentale, où les monuments qu'ont érigé nos prédécesseurs lointains et récents demeurent, les uns croulants et envahis d'une feuillaison irrévérencieuse, les autres fièrement dressés comme si les saisons du temps n'avaient eu aucune prise sur eux. Ce sont les monuments parmi lesquels nous vivons et pensons, même si nous le faisons parfois contre eux, ou malgré eux. On peut appeler cela déconstruction - ou peut-être anastylose, d'après le nom d'une approche quelque peu extrémiste de la prise en charge des temples préconisée par les architectes-colons et qui consistait à démanteler le monument avant de le reconstruire entièrement (en ajoutant des matériaux nouveaux où nécessaire), approche qui alliait admirablement dans un même geste destruction radicale et sauvegarde tout aussi radicale ${ }^{3}$. Car la reconstruction, si elle permet de protéger, comme archive et témoignage, telle image sculptée dans un mur du passé, construit toujours autre chose. En remontant des briques, on ne remonte

\footnotetext{
3. On trouvera un bref historique de cette méthode développée en Grèce pour être ensuite exportée aux Indes néerlandaises et en Indochine française dans Un siècle pour l'Asie: l'École française d'Extrême-Orient, 1898-2000, de Catherine Clémentin-Ojha et Pierre-Yves Manguin, 2001, p. 94.
} 
pas le temps. C'est plutôt le rapport que l'on entretient avec ces constructions qui importe désormais, la dynamique qui les emporte, et non pas un nouveau monument que l'on pourrait ériger à leur place.

Mais travailler sur l'Époque moyenne relève des études postcoloniales pour une autre raison : l'histoire du Cambodge, c'est-à-dire l'historiographie le concernant, est indissociable de la colonisation de l'Indochine et du protectorat français. On le sait, c'est grâce à la France que le Cambodge n'a pas été englouti par ses voisins rapaces à la fin du $\mathrm{XIX}^{\mathrm{e}}$ siècle. Mais non contente de sauver le Cambodge d'une disparition géographique et politique imminente, la France s'est donné une autre mission de sauvegarde. La même mission en sens inverse: sauver le Cambodge de la disparition, mais cette fois d'une disparition qui, aux yeux des Occidentaux, avait déjà eu lieu, en grande partie du moins, car il s'agit de la disparition de ce qui a déjà eu lieu: la disparition de la mémoire, d'une certaine mémoire dont les signes apparents sont très estimés à l'Ouest.

Ces « Mémoires du Cambodge » impliquent ainsi plus d'une histoire, plus d'une source, selon leur pluriel et le double sens de leur génitif. À qui une mémoire revient-elle? Nous ne pourrons jamais en être sûrs. Par conséquent, si cette thèse lui court après, c'est que la mémoire s'en va à travers elle en s'oubliant et en se souvenant, je l'espère, ailleurs et autrement. Ces questions de la mémoire, de ses missions, de sa transmission, de sa rémission parfois et de la vie ou de la survie culturelle sont particulièrement importantes pour le Cambodge aujourd'hui.

Or, les signes d'une volonté d'imposer une certaine mémoire à l'autre sont visibles, lisibles, notamment dans une grande partie des travaux sociopolitiques sur l'histoire récente du Cambodge, comme si en Occident on avait appris une fois pour toutes comment se souvenir afin que «plus jamais ça». Il s'agit d'une mémoire qui s'incarne dans un certain type de cérémonie, de monument aux morts, de muséologie, de texte et de discours sur l'histoire, grâce à ce qu'il faut bien appeler un travail de deuil (c'est-à-dire mémoire et oubli). Mais en dépit de la bonne conscience affichée, l'histoire du XXe siècle laisse penser au contraire que «nous» aussi avons peut-être à apprendre du Cambodge. C'est une source d'espoir au fond, un effet de ce qui pourrait être le visage positif de la mondialisation, malgré toutes les dissymétries meurtrières, notamment entre le monde industrialisé et le tiers monde, dont ladite mondialisation semble s'accommoder. 


\section{Labyrinthe, $n^{\circ} 23$}

On le répète depuis plus d'un siècle, ce sont les Français qui ont écrit l'histoire du Cambodge. Voilà jusqu'où peut aller le colonialisme d'un protectorat, quand un État détient jusqu'à l'histoire même d'un autre. Les Français étaient là, pour le meilleur et pour le pire. Et cette historiographie française sur le Cambodge racontait une certaine histoire. Une vieille histoire, certes, celle que l'on avait déjà récitée mutatis mutandis en Égypte, en Grèce, à Jérusalem et à Rome. Un brillant âge d'or, suivi d'une longue époque ténébreuse, était ainsi redécouvert par « nous », les auteurs de l'Histoire. Mais autour de la Méditerranée, l'Europe depuis la Renaissance, précisément, se retrouvait en quelque sorte en famille: les découvertes, aussi inattendues qu'elles aient pu l'être, s'apparentaient à des espèces d'anamnèses culturelles. Ces autres ignorés dont on était en train de faire la connaissance, trouvaient leur place en nous, comme s'ils avaient été nous-mêmes dans une vie antérieure.

Or, il n'en allait pas de même au Cambodge. Au départ, certes, on s'est essayé à domestiquer cette histoire, d'une certaine manière. En 1604, déjà, Gabriel Quiroga de San Antonio publie sa Breve y verdadera relacion de los successos del Reyno de Camboxa ${ }^{4}$ adressée à Philippe III d'Espagne pour le convaincre de l'intérêt de la conquête indochinoise. Il y apprend au roi que

la coutume de placer des serpents à la cime des mâts sur les chemins et celle des religieux d'entrer sept fois dans le chœur leur vient de certains juifs romains qui vécurent autrefois dans ce royaume. Il y a beaucoup de juifs dans le royaume de Chine: ce sont eux qui édifièrent au Cambodge la ville d'Angkor qui, ainsi que je l'ai dit, fut découverte en l'an 1570. Ils l'abandonnèrent quand ils émigrèrent en Chine $[\ldots]^{5}$.

Les Juifs, pour ce prêtre voyageur, ce sont en quelque sorte les autres de l'intérieur, «nos» autres, ou ceux de «nos» parents romains. L'Espagne s'est débarrassée, bien entendu, de cette mauvaise part d'ellemême, mais la présence de Juifs là-bas est un très bon signe:

Il y a au Cambodge de l'or, de l'argent, des pierreries, du plomb, de l'étain, du cuivre, de la soie, du coton, de l'encens, du benjoin, de la laque, de

\footnotetext{
4. Brève et Véridique Relation des événements du Cambodge, nouvelle édition avec traduction par A. Cabaton, Leroux, 1914.

5. Ibid., p. 100 (espagnol, p. 8-9).
} 
l'ivoire, du riz, des éléphants, des buffles, des chevaux, des vaches, des chèvres, des cerfs, des poules et des fruits aussi nombreux que savoureux. [...] une seule chose suffirait à démontrer ladite vérité, c'est l'affluence grande et continue des Japonais et des Chinois en ces royaumes. Ces étrangers sont comme les Juifs et même pires, ils ne vont jamais en terre stérile ou pauvre mais vivent et trafiquent toujours dans les pays où coulent le lait et le miel, où ils peuvent tirer profit. C'est une chose avérée que puisqu'ils font du commerce avec le royaume du Cambodge, c'est que ce royaume est très riche et procure de grands bénéfices. [...] ainsi sans aucun doute, ni exagération, Votre Majesté, en colonisant ce royaume ou les royaumes voisins, augmentera ses revenus et enrichira ses vassaux excessivement ${ }^{6}$.

Pour Gabriel Quiroga de San Antonio, les Juifs sont les colonisateurs de l'Occident, ses propres colons du dedans, ceux-là mêmes dont l'Espagne se serait affranchie, en effet, au moyen d'une " guerre d'indépendance » sous forme d'Inquisition. Renvoyés, ceux qui ont colonisé l'Occident deviennent les envoyés de celui-ci. C'est une première leçon quant au postcolonialisme: d'après San Antonio, le royaume d'Espagne, colonie décolonisée, n'a qu'à agir à l'exemple de ses anciens colonisateurs. C'est-à-dire de ses anciens Juifs, ou mieux encore de ces Juifs de l'Orient, « pires » que les Juifs, que sont les Japonais et les Chinois, ces « étrangers » asiatiques qui affluent, bien plus étrangers au Cambodge que les Espagnols, on l'aura compris. On les reconnaît à ce qu'ils ne s'établissent jamais dans un pays maudit, mais seulement là où « coulent le lait et le miel ». Dépêchons-nous d'y aller, dit San Antonio à son roi, au Cambodge, notre Terre promise ${ }^{7}$.

Quelque deux siècles et demi plus tard, quand les Français s'y installent enfin, on ne tient plus le même discours. Après les hésitations des premières heures, on reconnaît que ce sont les Khmers eux-mêmes qui ont réalisé les monuments d'Angkor. Mais si l'histoire du Cambodge ne se laisse pas vassaliser par celle de l'Europe, il n'y a qu'une solution, c'est de se l'approprier, en l'occurrence au moyen d'une grande mission de recherche. Il fallait le faire, sans doute, et les études khmères telles qu'on les connaît aujourd'hui n'existeraient

6. Ibid., p. 208 (espagnol, p. 78-79).

7. On imagine, d'ailleurs, que le plan de l'Escorial, expression architecturale du pouvoir souverain et impérial espagnol de l'époque, a été conçu comme une réplique du temple de Jérusalem. Voir Helen Rosenau, Vision of the Temple; the Image of the Temple of Jerusalem in Judaism and Christianity, Oresko Books Ltd., London, 1979, p. 94-5, ill. 97-100. 
même pas s'il n'y avait eu une poignée de surdoués français prêts à s'atteler à la tâche. Il fallait débroussailler, estamper, déchiffrer, étayer. Déconstruire et reconstruire. Il y avait tellement à faire sur la terre, on le sait, que l'on a très peu cherché sous elle. Beaucoup plus d'architectes que d'archéologues, pour aller vite, et cela jusqu'à l'heure actuelle. Et peu de temps pour s'occuper des vivants. La recherche ethnographique n'y a jamais joué de rôle que mineur et le grand projet anthropologique « français »- l'archivage, voire la reconstitution du « fait social total »- a été inconnu au Cambodge.

C'est ainsi que la vraie histoire du Cambodge s'est dessinée au fil des études, des traductions, des découvertes, selon un scénario passionnant et digne des meilleurs thrillers. Voir par exemple la séquence époustouflante qui se déroule en 1933 et dans laquelle G. Trouvé, le conservateur d'Angkor, trouve comme s'il y avait été prédestiné, la grande image du Bouddha en morceaux dans le puits central du temple du Bayon, trouvaille qui prouve, à l'étonnement général, la primauté bouddhique du règne de Jayavarman VII. Mais l'historiographie de cette histoire, sans en être moins vraie, était aussi surdéterminée, et ne pouvait pas ne pas participer, de gré ou de force, au discours colonialiste. Non seulement ce pays tombé dans une déchéance abyssale après un âge d'or glorieux fut sauvé par l'arrivée du Protecteur, mais il se trouve que cette histoire lui était due, qui restitue au Protégé une sorte de paradis perdu, retrouvé, et restauré perdu. Et dans cette histoire, ce roman ou ce film historique, l'Époque moyenne, effacée mais gardée comme telle, jouait un rôle clé depuis les coulisses où elle était assignée.

De ce point de vue, les Américains, qui débarquèrent dans ce pays un siècle plus tard, ou plutôt qui le survolèrent, furent terriblement plus efficaces: après le coup d'État de 1970, et l'établissement du régime proaméricain du Général Lon Nol, ils essayèrent de bombarder le Cambodge, comme ils l'avaient fait au Vietnam, «back to the Stone $A g e^{8} \gg$. À l'aide de tonnes d'explosifs largués en un véritable tapis de bombes, ils cherchèrent à créer eux-mêmes la désolation d'une sorte d'époque moyenne moderne qui, loin de « contenir » les ennemis idéologiques et de préparer le terrain pour l'arrivée du nouvel ordre américano-anticommuniste, précipita la suite que l'on connaît.

8. Selon le mot célèbre du Général C. LeMay. Voir B. Palmer, The 25-Year War: America's Role in Vietnam, New York, Touchstone, 1985, p. 29. 
Il se trouve que les Khmers rouges eux-mêmes ont fait leur version de cette historiographie dramatique qui tourne autour d'une Époque moyenne lamentable: ils ont essayé à leur tour de démanteler les structures de la civilisation telle qu'elle existait afin d'exécuter ensuite un «super grand bond en avant» [maha lot phloh maha achar], vers un nouveau monde, mais sans « population nouvelle » [pracheachun thmey] (terme qu'ils utilisaient pour désigner ceux des citadins «dégénérés » qui avaient échappé à leur contrôle jusqu'à 1975). Ce nouveau monde devait se bâtir en effet à partir d'une certaine tradition ancestrale que les Khmers rouges auraient redécouverte et rénovée. Voici ce qu'en disait Radio Phnom Penh le 12 juin 1975 dans son bulletin d'informations en langues étrangères:

En étudiant la civilisation, l'art et l'architecture du Cambodge, nous nous rendons compte que le peuple cambodgien a toujours été dur à la tâche, travailleur, créatif et talentueux [...]. En regardant Angkor, le temple d'Angkor Thom et ce qui l'entoure, nous sommes frappés par le fait que toute cette région était une grande ville traversée par des rues droites et des canaux dans le cadre d'un système magnifique [...] conçu sans la moindre erreur et réalisé avec la plus grande précision, le plus grand soin [...]. Cependant, notre nation et notre peuple cambodgiens ayant été régulièrement soumis à l'agression des impérialistes et du colonialisme ancien et nouveau, cette civilisation [...] s'éteignit [...]. [Aujourd'hui], sur la base de nos traditions, nous mêlons de nouveau tradition et science [...] conjuguant les traditions de notre pays à la science moderne. Notre peuple est en train d'édifier un nouveau Cambodge9.

Quand ils regardaient Angkor, les Khmers rouges ne voyaient justement pas le centre d'un puissant empire, mais seulement une " grande ville » dont la « civilisation » s'était éteinte sous la pression des « impérialistes et du colonialisme ancien et nouveau ». On pourrait même dire qu'une partie de ce qui était le plus abominable chez les Khmers rouges trouvait sa source, son inspiration ou du moins sa caution dans ce vieux trope de gloire perdue et retrouvée. Ils regardaient Angkor, mais ils ne voyaient pas que les apories de leur discours retournaient leur propre rhétorique contre eux pour en faire précisément des impérialistes et des colonisateurs qui disposaient à leur gré non seulement de l'histoire, qu'ils

9. Cité dans Elizabeth Becker, Les Larmes du Cambodge, Paris, La Cité, 1988, p. 190-1. 
refaçonnaient à leur convenance, mais surtout des milliers d'êtres humains dont ils avaient fait leurs autres dangereux et méprisables à juguler ou à éliminer sans pitié. Ou plutôt, c'est comme s'ils avaient senti la menace de ces apories et avaient cherché à s'auto-immuniser contre la rhétorique au moyen de la rhétorique, disant que le mélange qu'ils proposaient de «tradition et science »- eux qui avaient su conjuguer le passé lointain et le présent pour vaincre le futur sous la forme d'une nouvelle époque moyenne -, ce mélange, disaient-ils, venait lui-même de la tradition: "Sur la base de nos traditions, nous mêlons de nouveau tradition et science ». Ce que les Khmers rouges trouvaient qu'ils avaient trouvé, c'est que la conjugaison d'ancien et de nouveau était elle-même ancienne, et ils en jouèrent habilement pour se disculper d'avance de l'accusation d'impérialisme.

D'autre part, ils mélangeaient, selon la tradition, temple et ville. Angkor Thom, nom courant de la ville capitale construite au XII ${ }^{e}-\mathrm{XIII}{ }^{\mathrm{e}}$ siècle par Jayavarman VII, dernier grand roi angkorien, est aussi le nom populaire du Bayon, le grand temple érigé par ce même monarque au centre de sa ville. Cependant, grâce aux Khmers rouges, le peuple, leur peuple, était «en train d'édifier un nouveau Cambodge». Mais cela ne pouvait se faire tout seul. Il fallait d'abord que la capitale tombe. Le 17 avril 1975, le jour même où ils prirent Phnom Penh, ils commencèrent l'évacuation de tous ses habitants. La tâche fut accomplie efficacement en l'espace de trois jours. Phnom Penh fut dès lors une capitale abandonnée, tout à fait comme Angkor à l'Époque moyenne, abandonnée mais gardée, avec une sorte de fétichisme, dans un délabrement entretenu, comme s'ils avaient espéré la faire un jour visiter par les touristes du Nouveau Cambodge. Tout se passa en effet comme si la ville était en quelque sorte devenue le temple des Khmers rouges, un lieu sacré et mythique dont l'abandon était la garantie du nouvel âge à venir ${ }^{10}$.

Et ainsi de suite... Les derniers sauveurs du Cambodge - ceux qui viennent d'arriver - se plaignent à leur tour de la perte abominable de l'histoire: l'histoire est perdue et c'est l'histoire elle-même qui a opéré cette perte. Ses élites quasi exterminées, ses archives comme ses structures sociales détruites, le Cambodge, dit-on, a perdu jusqu'à sa mémoire:

10. Notons que le Prince Sihanouk sous le Sangkum Reastr Niyum après l'Indépendance, et notamment à travers ses projets de grands travaux, a ranimé, ou prolongé en la transformant, une grande tradition de constructions royales: depuis le monument d'Indépendance et le Stade olympique (temple-montagnes) de la capitale jusqu'aux usines, hôpitaux, etc., dans les provinces. 
encore une fois. Le monde sonne l'alarme du refoulement voire de l'oubli, avec la régularité d'une horloge, dès que l'on actionne le ressort « Cambodge ». À titre d'exemple, citons Le Monde du 17 avril 199811: «La mémoire et le traumatisme [...] sont provisoirement occultés [...]. Le Cambodge est un pays [...] sans monuments aux morts», y déclare J.-Cl. Pomonti sans douter de rien, avant de demander, " Amnésie? Refoulement?», questions évidemment « rhétoriques». Au moyen de livres scolaires et de tribunaux, sans oublier la restauration du site d'Angkor - immense chantier de reconstruction de monuments aux morts, justement -, on se donne la mission de restituer sa mémoire à un Cambodge oublieux.

Or, ce sont souvent des chercheurs ne sachant eux-mêmes rien de l'Époque moyenne qui se lamentent le plus amèrement des défaillances mnésiques ou mémoriales des Khmers modernes. On n'a pas nécessairement tort de se lamenter, mais pour traiter de ces questions de la mémoire, questions qui nous concernent au plus haut degré, il nous faut en appeler à toutes nos forces, les rappeler à nous et nous les rappeler. D'abord parce que nous sommes loin de savoir ce qu'est ou pourrait être la mémoire. Sans doute n'est-elle jamais simplement là. En Occident surtout, c'est à peine si les douloureuses histoires du $\mathrm{XX}^{\mathrm{e}}$ siècle ont commencé à remuer les cendres dont elle se voile.

En effet, on peut considérer que le Cambodge, certes changeant, de la période moyenne - où s'élève déjà, comme sur un gouffre, un royaume bouddhiste theravadin intégrant de manière complexe un passé brahmanique et fourmillant de croyances animistes - a réuni pour la première fois ce que l'on pourrait appeler les traits marquants du Cambodge tel qu'il se présente de nos jours. C'est-à-dire qu'en étudiant le Cambodge moderne, on lui fait tort si l'on passe sous silence cette partie de son passé. Autre problématique à classer sous la rubrique du postcolonialisme. Peut-on se permettre, alors qu'on s'interroge sur l'histoire récente du Cambodge, de faire l'économie de ces restes peu reluisants de plusieurs siècles d'Époque moyenne, ce qui se fait souvent, et notamment dans les sciences politiques? Or, caractérisée précisément par le manque de données concrètes, cette période est la moins connue de toute l'histoire khmère. Je dirais pour commencer que ce vide archival lui-même et en tant que tel, en dehors de ce qu'il peut cacher ou révéler d'une histoire

11. « Ces Khmers qui ne sont plus rouges », p. 12. 


\section{Labyrinthe, $n^{\circ} 23$}

événementielle, pèse toujours et lourdement sur le Cambodge moderne, sur tous ses aspects sociaux et culturels. L'historien n'est jamais neutre, on le sait, dans une telle histoire. Là encore, c'est une question de mémoire. De la mémoire du chercheur ou du scientifique autant que de son sujet ou objet d'études. Car l'un n'est pas sans rapport avec l'autre. 